\title{
Effect of high pressures and high temperatures on the structure of nanostructured titanium monoxide
}

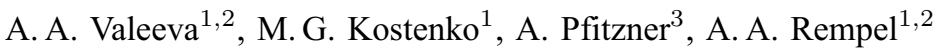 \\ ${ }^{1}$ Institute of Solid State Chemistry UB RAS, 91, Pervomaiskaya st., Ekaterinburg, 620990, Russia \\ ${ }^{2}$ Ural Federal University named after the first President of Russia B. N. Eltsin, \\ 91, Mira st., Ekaterinburg, 620002, Russia \\ ${ }^{3}$ Institute of Inorganic Chemistry, Regensburg University, Regensburg, Germany \\ anibla_v@mail.ru
}

PACS 61.72.Dd, 61.72.Bb, 64.70.Nd, 71.20.Ps

DOI 10.17586/2220-8054-2018-9-4-544-548

\begin{abstract}
The structure of nanostructured titanium monoxide $\mathrm{TiO}_{0.98}$ containing structural vacancies in two sublattices simultaneously has been modified via thermobaric annealing. Analysis of the experimental data on thermobaric synthesis of nanostructured $\mathrm{TiO}_{0.98}$ with cubic $B 1$ type structure at temperatures $573-2273 \mathrm{~K}$ and pressure $6 \mathrm{GPa}$ revealed that a transition from the cubic $B 1$ (sp. gr. $F m \overline{3} m$ ) phase to the trigonal $\mathrm{Ti}_{2} \mathrm{O}_{3}$ (sp. gr. $R \overline{3} c$ ) phase takes place in the nanostructured monoxide as a result of high pressures and high temperatures. The first-principle calculations of the cohesive energy and electronic structure show that the trigonal phase with space group $R \overline{3} c$ is energetically favorable compared to the cubic phase of the same composition $\mathrm{TiO}_{3 / 2}$ and the orthorhombic ordered $\mathrm{Ti}_{2} \mathrm{O}_{3}$ (sp. gr. Immm) phase.
\end{abstract}

Keywords: nanostructured titanium monoxide, structural vacancy, nonstoichiometry, high pressure, high temperature, phase transition, electronic structure.

Received: 25 May 2018

Revised: 12 July 2018

\section{Introduction}

Oxides, carbides and nitrides of transition metals of IV - V groups of the periodic table having $B 1$ type crystal structure feature wide homogeneity regions due to an anomalously high concentration of structural vacancies crystal lattice sites which are not occupied by atoms [1,2]. Vacancies are associated not only with nonstoichiometry, but also with short- and long-range order effects allowing a great number of various phases and structural modifications of a compound to be obtained while retaining the type of its basis crystal structure [3-8]. This peculiarity is of importance in terms of the topical problem of designing materials with pre-defined properties.

Titianium monoxide is a unique compound with a wide homogeneity region $\left(\mathrm{TiO}_{0.7}-\mathrm{TiO}_{1.3}\right)$ and an anomalously high (to 30 at. \%) concentration of vacancies simultaneously in the metal and nonmetal sublattices of $B 1$ structure [8-13]. The experimental works performed earlier on samples of microcrystalline titanium monoxide showed that electron irradiation [14,15] and the treatment by pressure [16-19] or heat [8-12] lead to the modification of the structure and properties by changing the concentration of the vacancies or the way of their arrangement in the structure. In the nanostate, in addition to point defects (structural vacancies), two-dimensional defects (crystal surface) should also be taken into consideration. The interference of these two types of defects extends the variety of possible structural modifications and the range of controllable properties [20-23]. In particular, while hightemperature annealing of microcrystalline titanium monoxide gives rise to the monoclinic phase $\mathrm{Ti}_{5} \mathrm{O}_{5}$ [10-12], in the experiment on nanostructured samples a $\mathrm{Ti}_{9} \mathrm{O}_{10}$ phase was observed whose structure is not typical of microcrystalline state [24]. The aim of this work is to study the behavior of the structure of nanostructured titanium monoxide under high pressure and high temperature.

\section{Experimental}

The microcrystals of titanium monoxide $\mathrm{TiO}_{y}$ with the average size of $\sim 25 \mu \mathrm{m}$ have been synthesized by the solid phase sintering from a mixture of titanium $\mathrm{Ti}$ and titanium dioxide $\mathrm{TiO}_{2}$ powders in vacuum $10^{-3} \mathrm{~Pa}$ at $1770 \mathrm{~K}$. In order to achieve a disordered state of titanium dioxide, the evacuated $\left(10^{-3} \mathrm{~Pa}\right)$ quartz ampoules with samples were annealed for $3 \mathrm{~h}$ at $1330 \mathrm{~K}$, whereupon they were dropped into the water, the quenching rate was $\sim 200 \mathrm{~K} / \mathrm{s}$. According to the XRD data, the microcrystalline powder contained reflections only of the cubic phase with $B 1$ structure (sp. gr. $F m \overline{3} m$ ) with a disordered arrangement of vacancies in sublattices. A multi-parameter certification of the samples was carried out with the use of chemical, spectral, pycnometer, X-ray diffraction and 
X-ray structure analysis methods. The heat treatment method for obtaining the disordered and ordered states and the results of attestation of the synthesized samples are described in detail in work [12].

Nanocrystals of titanium monoxide were produced by high-energy milling in a Retsch PM 200 planetary ball mill using $\mathrm{Y}_{2} \mathrm{O}_{3}$-stabilized $\mathrm{ZrO}_{2}$ grinding media and vials. The ball-to-powder weight ratio in our experiments was 10:1. The milling liquid used was isopropanol. The high-energy milling conditions were as follows: milling time of $15,30,60,120,240$, and $480 \mathrm{~min}$; rotation direction reversed every $15 \mathrm{~min}$; interval between direction reversals, $5 \mathrm{~s}$; rotation speed of the disk supporting the grinding vials, $500 \mathrm{rpm}$ [22].

The XRD studies of $\mathrm{TiO}_{y}$ were carried out with a STOE STADI P transmission diffractometer equipped with a linear PSD, a Ge-monochromator, and a Mythen $1 \mathrm{~K}$ detector at $293 \mathrm{~K}$. The measurements were performed using $\mathrm{CuK} \alpha_{1}$ radiation, the $2 \theta$ range was $10-116^{\circ}$ with a step-width of $0.015^{\circ}$. Polycrystalline silicon $(a=543.07 \mathrm{pm})$ was used as external standard. The XRD patterns were measured both in a short run and a longer run with better statistics. Four XRD powder patterns were summed up for better statistics and were used for a full profile analysis. The error of the lattice constant determination was about $1 \mathrm{pm}$. The phase analysis of the XRD pattern was performed using the Powder Cell 2.4 program. For phase identification the powder standards database ICDD PDF2 (ICDD, USA, Release 2009) was used. There are small crystalline peaks around $2 \theta=36.7,41.5$ and 54.2 degree in the initial sample. According to phase analysis, the impurity lines on the XRD pattern are from an unidentified compound.

In addition to reducing particle size, that is, comminuting, milling produces internal lattice strain in the resulting nanoparticles. The diameter of nanocrystals $D$ was determined from the broadening of X-ray diffraction reflections by the Williamson-Hall method $[25,26]$. Owing to the fragmentation of ordered titanium monoxides with near-stoichiometric compositions, we obtained nanoparticles $20 \pm 10 \mathrm{~nm}$ in size, with the lowest lattice strain at a level of $0.3 \%$. The fragmentation of disordered titanium monoxides with both substoichiometric and superstoichiometric compositions resulted in roughly the same particle size, about $40 \pm 10 \mathrm{~nm}$, but the lattice strain was three times higher. Analysis of X-ray diffraction patterns showed that high-energy milling led to disordering and reduced the degree of long-range order. The techniques used to prepare nanocrystals by high-energy milling, analysis of X-ray diffraction patterns, SEM and TEM pictures, calculation of the coherent scattering region sizes were described elsewhere [22,24].

High-pressure experiments were performed on a modified high-pressure plant (Sack \& KiesselBach 1041, Germany). The nanostructured titanium monoxide powder was placed into a cylindrical boron nitride capsule; then the capsule was covered with pyrophyllite $\mathrm{Al}_{2}(\mathrm{OH})_{2}\left(\mathrm{Si}_{2} \mathrm{O}_{5}\right)_{2}$ serving as a medium for pressure transfer. After that, the upper and lower parts of the capsule were covered with graphite lids. Initially, the pressure was increased to $6 \mathrm{GPa}$ over $60 \mathrm{sec}$, and then the temperature was raised, also over $60 \mathrm{sec}$. In this study, the experiments were carried out at 573, 773, 1273, 1773 and $2273 \mathrm{~K}$. The pressure and temperature were held constant for 60 min, then the temperature in the cell was rapidly decreased to ambient temperature at the rate of $\sim 200 \mathrm{~K} / \mathrm{sec}$ at approximately constant pressure, and after that, the pressure was released to atmospheric pressure during several seconds. The experiments were repeated 2-3 times under the same conditions, and according to the XRD data, the reproducibility of the results was observed.

\section{Results and discussion}

The results of X-ray diffraction experiments on samples subjected to thermobaric annealing are presented in Table 1 and Fig. 1. The microcrystalline state, according to [16-19], is characterized by large lattice period values, $418-421 \mathrm{pm}$. The temperature dependence of the lattice period for the nanostructured state is not monotonic: in the temperature interval from 298 to $773 \mathrm{~K}$ the lattice period increases from 417.6 to $417.82 \mathrm{pm}$. At $1273 \mathrm{~K}$, the lattice period decreases to $417.5 \mathrm{pm}$ and at $1773 \mathrm{~K}$ it reaches the value of $416.07 \mathrm{pm}$. At $2273 \mathrm{~K}$, the lattice period increases to $417.54 \mathrm{pm}$, which is somewhat smaller than that observed at $298 \mathrm{~K}$. The temperature range, over which the lattice period of the basis structure increases monotonously, corresponds to the sole initial disordered cubic phase. At temperatures above $1273 \mathrm{~K}$, the system becomes two-phase. A trigonal phase $\mathrm{Ti}_{2} \mathrm{O}_{3}$ (sp. gr. $R \overline{3} c$ ) is formed, whose structure is not a $B 1$ derivative and contains no vacancies.

The first-principle calculations of the cohesion energy and electronic structure, performed by the technique described in [24] with the use of code [27], reveal that the trigonal phase with the $R \overline{3} c$ space group is energetically advantageous compared with the disordered cubic phase of the same $\mathrm{TiO}_{3 / 2}$ composition and with the orthorhombic ordered phase $\mathrm{Ti}_{2} \mathrm{O}_{3}$ (sp. gr. Immm) [28] having a B1-derived structure. The calculated cohesive energy of the phase with $R \overline{3} c$ space group is $-17.76 \mathrm{eV}$ vs. $-16.21 \pm 0.04 \mathrm{eV}$ for the disordered cubic phase and $-17.25 \mathrm{eV}$ for the orthorhombic ordered phase with space group Immm. Fig. 2 displays the density of electronic states calculated for three alternative structures of the $\mathrm{TiO}_{3 / 2}$ composition. All of them possess metallic conduction; the largest density of states at the Fermi level was observed for the orthorhombic model [28]. The trigonal phase observed 
TABLE 1. The conditions of thermobaric treatment of nanostructured titanium monoxide $\mathrm{TiO}_{0.98}$ and the XPD results after treatment

\begin{tabular}{|c|c|c|c|c|c|c|}
\hline \multirow{2}{*}{$T, P$} & \multicolumn{2}{|c|}{$\mathrm{TiO}_{y}$ (sp. gr. $\mathrm{Fm} \overline{3} m$ ) } & \multicolumn{3}{c|}{$\mathrm{Ti}_{2} \mathrm{O}_{3}$ (sp.gr. $R \overline{3} c$ ) } \\
\cline { 2 - 7 } & $\begin{array}{c}\text { Lattice } \\
\text { period } \\
a_{B 1}, \mathrm{pm}\end{array}$ & $\begin{array}{c}C_{T i O y}, \\
\text { mass.\% }\end{array}$ & $\begin{array}{c}\text { Crystal } \\
\text { size D, nm }\end{array}$ & $\begin{array}{c}\text { Lattice } \\
\text { period } \\
a_{B 1}, \mathrm{pm}\end{array}$ & $\begin{array}{c}C_{T i 2 O 3}, \\
\text { mass. \% }\end{array}$ & $\begin{array}{c}\text { Crystal } \\
\text { size D, nm }\end{array}$ \\
\hline \hline $298 \mathrm{~K}$ & 417.60 & 100 & 40 & - & - & - \\
\hline $\begin{array}{c}573 \mathrm{~K}, \\
6 \mathrm{GPa}\end{array}$ & 417.63 & 100 & 42 & - & - & - \\
\hline $\begin{array}{c}773 \mathrm{~K}, \\
6 \mathrm{GPa}\end{array}$ & 417.82 & 100 & 50 & - & - & - \\
\hline $\begin{array}{c}1273 \mathrm{~K}, \\
6 \mathrm{GPa}\end{array}$ & 417.05 & 70 & 50 & $\begin{array}{c}511.4, \\
1379.0\end{array}$ & 30 & 75 \\
\hline $\begin{array}{c}1773 \mathrm{~K}, \\
6 \mathrm{GPa}\end{array}$ & 416.07 & 60 & 50 & $\begin{array}{c}511.7, \\
1379.9\end{array}$ & 40 & 80 \\
\hline $\begin{array}{c}2273 \mathrm{~K}, \\
6 \mathrm{GPa}\end{array}$ & 417.54 & 30 & 50 & $\begin{array}{c}512.3, \\
1378.7\end{array}$ & 70 & 80 \\
\hline
\end{tabular}

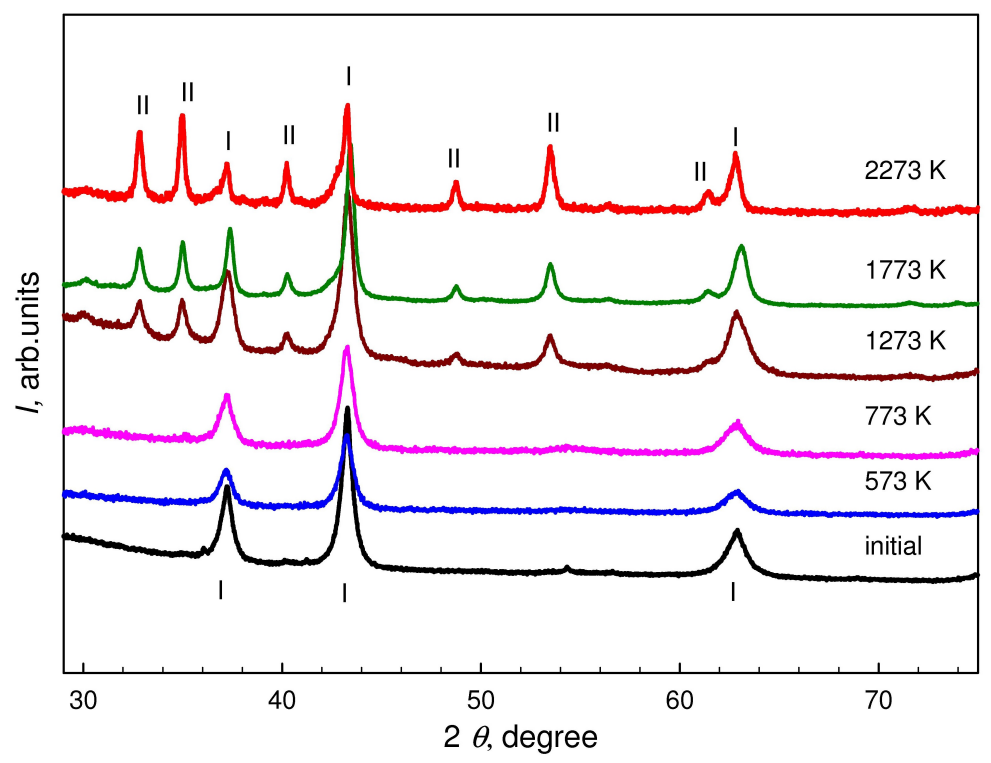

FIG. 1. The experimental X-ray diffraction patterns of $\mathrm{TiO}_{0.98}$ powders: initial nanostructured powder; after thermobaric treatment at $6 \mathrm{GPa}$ and 573, 773, 1273, 1773, $2273 \mathrm{~K}$ (phases: I $\mathrm{TiO}_{y}$ (sp. gr. $F m \overline{3} m$ ), II $-\mathrm{Ti}_{2} \mathrm{O}_{3}$ (sp. gr. $\left.R \overline{3} c\right)$ )

in the experiment was characterized by a much wider $p-d$ gap between the valence band and the conduction band compared with the $p-d$ gap of $B 1$ derived structures.

The nonmonotonic character of the temperature dependence of the lattice period observed in the experiment is likely to be due to the isolation of the trigonal phase $\mathrm{Ti}_{2} \mathrm{O}_{3}$, whose composition $\left(\mathrm{TiO}_{3 / 2}\right)$ differs from the composition of the initial disordered cubic phase $\mathrm{TiO}_{0.98}$. In order to retain the initial ratio of components in the two-phase system, the composition of the disordered cubic phase should be shifted into the substoichiometric region. However, for the microcrystalline phase under atmospheric pressure this would mean a lattice period enhancement [29], whereas in our case the lattice period decreases. This suggests a different mechanism of variation in the composition and vacancy concentration compared with the microcrystalline state under atmospheric pressure. Note also that the nanostate features smaller lattice period values compared with the microcrystalline state, which agrees with the predictions of theoretical calculations [30]. 


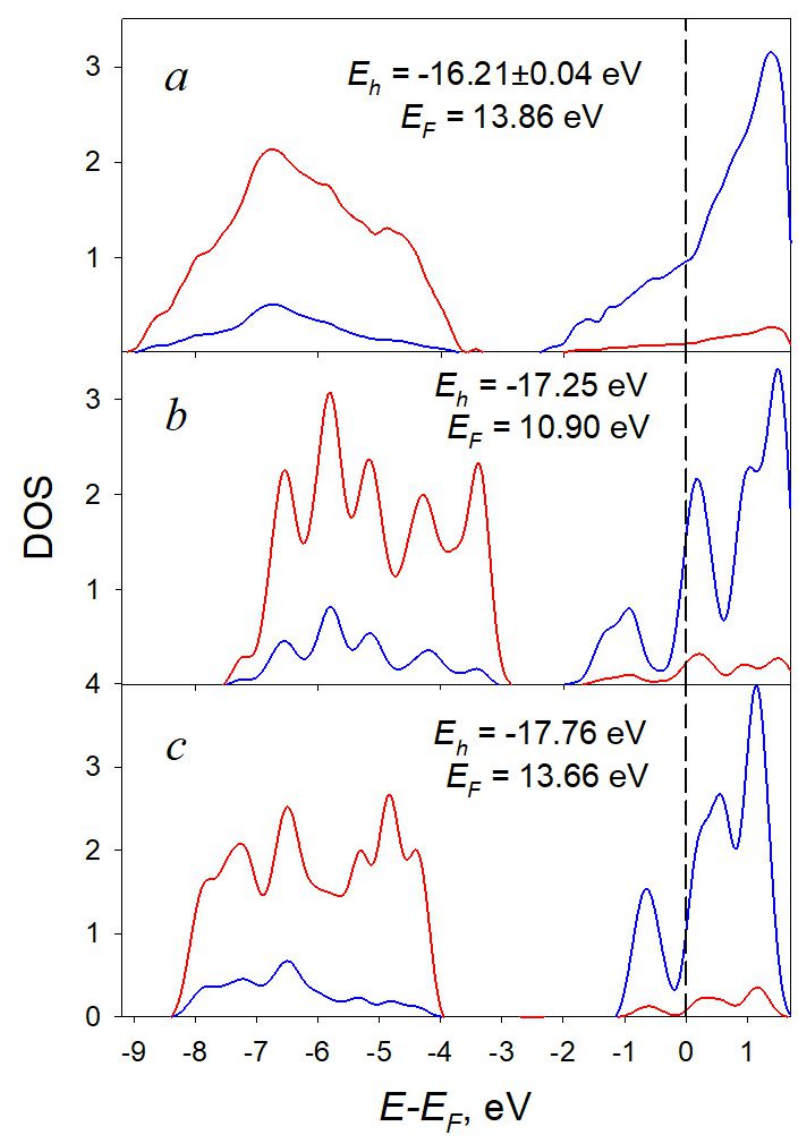

FIG. 2. The density of $\mathrm{O} 2 p$ (red lines) and Ti $3 d$ (blue lines) electronic states calculated for the disordered cubic phase $\mathrm{TiO}_{3 / 2}$ (sp. gr. $F m \overline{3} m$ ), the orthorhombic ordered phase $\mathrm{Ti}_{2} \mathrm{O}_{3}$ (sp. gr. Immm), as well as for the trigonal phase $\mathrm{Ti}_{2} \mathrm{O}_{3}$ (sp. gr. $R \overline{3} \mathrm{c}$ ) observed in the experiment

\section{Conclusion}

In the present work, the nanostructured $\mathrm{TiO}_{0.98}$ powder, which contained structural vacancies in two sublattices simultaneously, was subjected to high pressures up to $60 \mathrm{kbar}$ and high temperatures up to $2273 \mathrm{~K}$. The X-ray powder diffraction revealed a change of the structure from cubic $\mathrm{TiO}_{y}$ phase (sp. gr. $F m \overline{3} m$ ) to the trigonal phase $\mathrm{Ti}_{2} \mathrm{O}_{3}$ (sp. gr. $R \overline{3} c$ ) after applying the high pressure in combination with the high temperature. According to first-principle calculations, the trigonal $\mathrm{Ti}_{2} \mathrm{O}_{3}$ (sp. gr. $R \overline{3} c$ ) phase observed in the experiment is energetically favorable compared to the cubic phase of the same $\mathrm{TiO}_{3 / 2}$ composition and the orthorhombic-ordered $\mathrm{Ti}_{2} \mathrm{O}_{3}(\mathrm{sp}$. gr. Immm) phase and is characterized by a much wider $p-d$ gap between the valence band and the conduction band compared with the $p-d$ gap of structures derived from $B 1$ structure. The purposeful synthesis of materials based on particles of various stoichiometry, crystal size and dimension with pre-defined properties and structure gives enhanced functionality and provides comprehensive control of the settings.

\section{Acknowledgements}

We are grateful to Dr. M. Andratschke, F. Rau, and U. Schiessl (Institute of Inorganic Chemistry, Regensburg University) for their help during the experiments.

The work was carried out at the Institute of Solid State Chemistry UB RAS with financial support from the Russian Science Foundation (project 14-23-00025). The electronic structure calculations were performed on "Uran" supercomputer at the IMM UB RAS. 


\section{References}

[1] Gusev A.I., Rempel A.A., Magerl A.J. Disorder and Order in Strongly Nonstoichiometric Compounds: Transition Metal Carbides, Nitrides and Oxides. Springer: Berlin-Heidelberg-New York, 2001.

[2] Rempel A.A., Valeeva A.A. Thermodynamics of atomic ordering in nonstoichiometric transition metal monoxides. Mend. Communication, 2010, 20, P. 101-103.

[3] Kostenko M.G., Valeeva A.A., Rempel A.A. Relationship between short- and long-range orders in nonstoichiometric titanium monoxide $\mathrm{TiO}_{y}$. JETP, 2010, 111, P. 786-795.

[4] Kostenko M.G., Rempel A.A., Lukoyanov A.V. Internal energy and parameters of the order-disorder phase transition in titanium monoxide $\mathrm{TiO}_{y}$. JETP, 2013, 116, P. 945-951.

[5] Kostenko M.G., Rempel A.A. Electrostatic stabilization of an ordered phase in titanium monoxide. Phys. Sol. St., 2010, 52, P. 776-780.

[6] Kostenko M.G., Rempel A.A. Probabilities of octahedral clusters depending on long-range order parameters and composition in nonstoichiometric titanium monoxide $\mathrm{TiO}_{y}$. JETP, 2012, 115, P. 99-107.

[7] Kostenko M.G., Valeeva A.A., Rempel A.A. Octahedral clusters in various phases of nonstoichiometric titanium monoxide. Mend. Communication, 2012, 22, P. 245-247.

[8] Valeeva A.A., Rempel S.V., Schroettner H., Rempel A.A. Influence of the degree of order and nonstoichiometry on the microstructure and microhardness of titanium monoxide. Inorganic materials, 2017, 53, P. 1174-1179.

[9] Andersson S., Collen B., Kuylenstierna U., Magneli A. Phase-analysis studies on the titanium-oxygen syste. Acta Chem. Scand., 1957, 11, P. $1641-1652$.

[10] Watanabe D., Castles J.R., Jostsons A., Marlin A.S. Ordered structure of titanium oxide. Nature, 1966, 210, P. 934-936.

[11] Banus M.D., Reed T.B. Structural, electrical and magnetic properties of vacancy stabilized cubic TiO and VO, in: L. Eyring, M.O. Keeffe (Eds.), The Chemistry of Extended Defects in Non-Metallic Solids, Amsterdam-London: North-Holland Publ., 1970, P. 488-521.

[12] Valeeva A.A., Rempel A.A., Gusev A.I. Ordering of cubic titanium monoxide into monoclinic Ti ${ }_{5} \mathrm{O}_{5}$. Inorganic materials, 2001, 37, P. 603-612.

[13] Gusev A.I., Valeeva A.A. Diffraction of electrons in the Cubic Ti5O5 superstructure of titanium monoxide. JETP Letters, 2012, 96, P. 364-369.

[14] Rempel A.A., Renterghem W.V., et al. In situ disordering of monoclinic titanium monoxide $\mathrm{Ti}_{5} \mathrm{O}_{5}$ studied by transmission electron microscope TE.M. Scientific reports, 2017, 7, 10769.

[15] Guo C., Jia S., et al. Orientation domains in vacancy-ordered titanium monoxide. Acta Cryst. B, 2013,69 , P. 589-594.

[16] Kostenko M.G, Valeeva A.A., Rempel A.A. Effect of high pressure on the period of the bais lattice and concentration of vacancies in titanium monoxide TiO. JETP Lett., 2017, 106, P. 354-357.

[17] Valeeva A.A., Rempel A.A., Pfitzner A. Elimination of vacancies in titanium monoxide under high pressure in combination with high temperature. Journal of Monatshefte für Chemie - Chemical Monthly, 2015, 146, P. 1205-1209.

[18] Taylor A., Doyle N.G. Vacancy filling in titanium monoxide and similar semi-metals, In: The chemistry of extended defects in non-metallic solids, Eds by L. Eyring, M. O’Keefe. Amsterdam-London: North-Holland Publ., 1970, P. 523-540.

[19] Fujimura T., Iwasaki H., et al. Structure changes in vacancy-rich titanium monoxide at high pressures and high temperatures. High pressure research, 1989, 1, P. 213-224.

[20] Valeeva A.A., Nazarova S.Z., Rempel A.A. Influence of Particle Size, Stoichiometry, and Degree of Long-Range Order on Magnetic Susceptibility of Titanium Monoxide. Physics of the Solid State, 2015, 58, P. 771-778.

[21] Rempel A.A. Hybrid nanoparticles based on sulfides, oxides, and carbides. Russ. Chem. Bull., 2013, 4, P. 857-868.

[22] Valeeva A.A., Petrovykh K.A., Schroettner H., Rempel A.A. Effect of stoichiometry on the size of titanium monoxide nanoparticles produced by fragmentation. Inorganic Materials, 2015, 51, P. 1132-1137.

[23] Rempel S.V., Valeeva A.A., et al. Vacuum-made nanocomposite of low-temperature hydroxyapatite and hard nonstoichiometric titanium monoxide with enhanced mechanical properties. Mend. Communication, 2016, 26, P. 543-545.

[24] Valeeva A.A., Kostenko M.G., et al. A new $\mathrm{Ti}_{9} \mathrm{O}_{10}$ nanophase prepared by heat-treating nonstoichiometric milled TiO $y$ nanopowder. Inorganic Materials, 2018, 54, P. 568-574.

[25] Hall W.H. X-ray line broadening in metals. Proc. Phys. Soc. London. Sect. A, 1949, 62, P. 741-743.

[26] Hall W.H., Williamson G.K. The diffraction pattern of cold worked metals: I. The nature of extinction. Proc. Phys. Soc. London B, 1954, 64, P. 937-946.

[27] Giannozzi P., Baroni S., et al. Quantum Espresso: a modular and open-source software project for quantum simulations of materials. Journal of Physics. Condensed Matter, 2009, 21, 395502 (19 pp.)

[28] Gusev A.I. Ordered orthorhombic phases of titanium monoxide. JETP Letters, 2001, 74, P. 91-95.

[29] Banus M.D., Reed T.B., Strauss A.J. Electrical and magnetic properties of TiO and VO. Physical Review B, 1972, 5, P. $2775-2784$.

[30] Popov I.S., Enyashin A.N., Rempel A.A. Size dependent content of structural vacancies within TiO nanoparticles: Quantum-chemical DFTB study, Superlattices and Microstructures, 2018, 113, P. 459-465. 